\title{
CONGENITAL ABSENCE OF THE ABDOMINAL MUSCLES WITH ASSOCIATED ABNORMALITIES OF THE GENITO-URINARY TRACT
}

BY

\author{
PETER ROBERTS \\ From the Royal Hospital, Wolverhampton
}

(RECEIVED FOR PUBLICATION FEBRUARY 13, 1956)

The syndrome of congenital absence of the abdominal muscles associated with other abnormalities, chiefly of the genito-urinary tract, has received little attention in this country. Housden in 1934 reviewed 33 cases reported up to that time and added a further case of his own. Hill's (1947) case does not appear to have been a true example of this condition as there was a large defect of the anterior abdominal wall covered only by peritoneum.

Of the four cases presented here, three are examples of the condition while the fourth patient has suffered from recurrent chest infections.

\section{Case Reports}

Case 1. M.K., a boy, is the third child of healthy parents. Both siblings are boys and both are healthy. He was referred to hospital in September, 1953, at the age of 2 years and 1 month because of enlargement of the abdomen, which had been present since birth. The birth history and development up to that time was normal. On examination he was found to have a prominent abdomen with marked transverse creases. The abdomen bulged laterally, particularly to the right and especially noticeably on straining. The oblique and transversal:s muscles were absent. The recti were present but deficient and divaricated. The liver and spleen were both palpable. The testes were bilaterally undescended. The blood pressure was $110 / 60 \mathrm{~mm}$. Hg. The urine was found to be infected with Staphylococcus aureus. An intravenous pyelogram showed dilatation and elongation of both ureters but both kidneys appeared normal. A retrograde cystogram showed a thin bladder of abnormal shape with marked trabeculation.

The urinary infection was cleared with 'gantrisin' and chloramphenicol. The child was fitted with an abdominal corset and his mother instructed to make him urinate at regular intervals.

He remained well but was re-admitted to hospital in November, 1955, for re-assessment of the urinary tract. At that time he was a happy, well-nourished boy. He was passing urine normally and there was no incontinence. Physical examination showed no change. His urine was sterile and his blood urea level $43 \mathrm{mg} .{ }^{\circ}$.
An intravenous pyelogram showed dilatation of the ureters, more marked on the left side, but no evidence of hydronephrosis. A retrograde cystogram showed an obstruction of the bladder neck (Fig. 1).

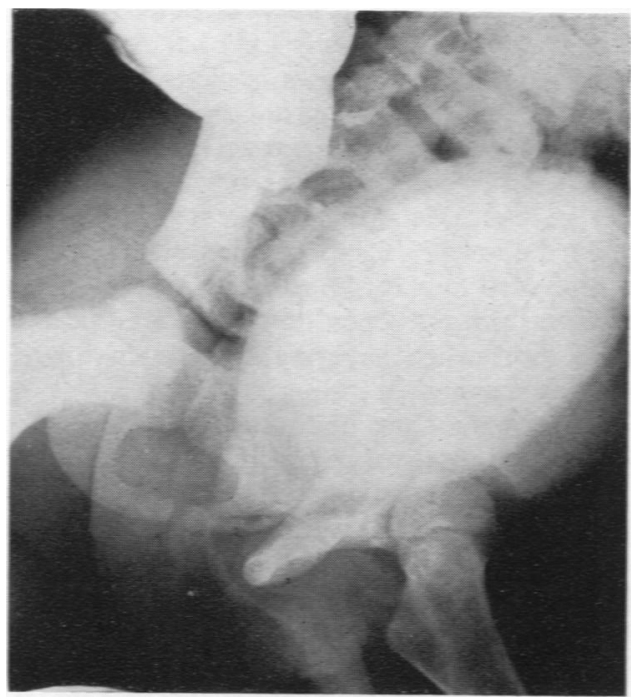

Fig. 1.-Retrograde cystogram in Case 1 showing a large bladder with dilatation of the posterior urethra.

Case 2. A.C. was admitted to hospital in March, 1952, at the age of 6 weeks. He was delivered by forceps following a full-time pregnancy. Birth weight was $8 \frac{1}{2} \mathrm{lb}$. Examination showed marked transverse creasing of the skin of the abdomen (Fig. 2). The oblique and transversalis muscles were absent. The left rectus was better felt than the right and it was noticed that in the supine position the abdomen tended to sag to the right. The abdominal organs were all easily palpable and the bladder was distended half-way up to the umbilicus. Blood pressure was $85 /$ ? $\mathrm{mm}$. Hg. The urine was sterile. The blood urea level was $29 \mathrm{mg}$. \%. A subcutaneous pyelogram was unsuccessful. A retrograde cystogram showed an enlarged bladder but no reflux to the 


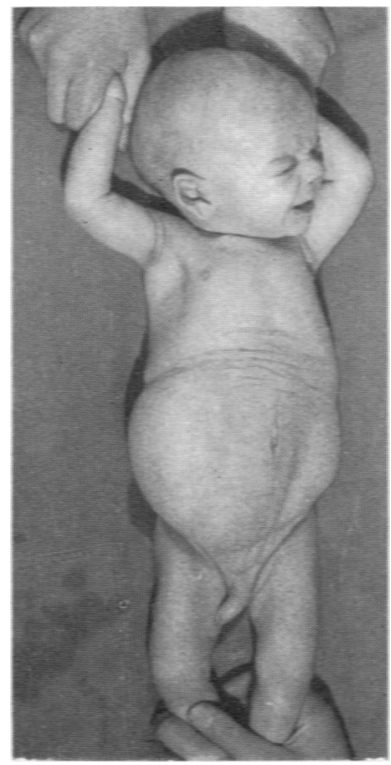

Fig. 2.-Case 2 showing typical appearance of the abdominal wall with transverse creasing and bulging flanks.

ureters. The baby was fitted with an abdominal binder and discharged home.

He remained well but was re-admitted in May, 1955. The abdominal wall was bulging grossly. The bladder was palpable just above the symphysis pubis. Both testes were bilaterally undescended. Blood pressure was $90 / 55 \mathrm{~mm}$. $\mathrm{Hg}$. The blood urea level was $16 \mathrm{mg}$. ${ }^{\circ}$. A subcutaneous pyelogram showed bilateral gross hydroureter and hydronephrosis. A micturating cystogram was attempted but the patient refused to cooperate. He was again discharged home wearing his corset.

Case 3. K.M. was the second child of healthy parents, born following a normal pregnancy and weighing $8 \mathrm{lb}$. $4 \mathrm{oz}$. Delivery was difficult owing to abdominal enlargement and for this reason the infant was referred to hospital. The infant had not passed urine or meconium before admission.

On admission to hospital, six hours after birth, the abdomen was grossly enlarged. The anterior abdominal wall was thin and wrinkled and in both flanks there were visible distended, lobulated masses (Fig. 3), which resembled intestine but were considered to be enormously dilated and hypertrophied ureters. The bladder was palpable just below the umbilicus. The liver, spleen and kidneys were all palpable. The penis was abnormal in that the foreskin was excessively long with a small external opening. A small quantity of urine was present within the foreskin. Both testes were undescended. There was also a minor degree of talipes equino-varus, but no other abnormalities were found.

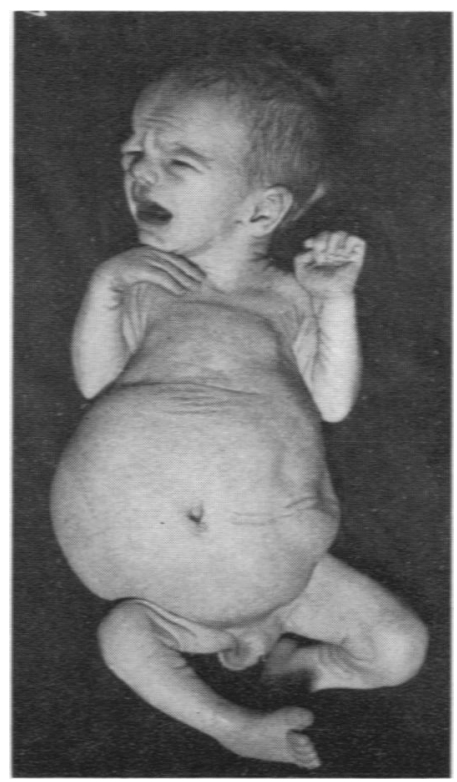

FiG. 3.- Case 3 showing appearance of the abdominal wall and the outline of the dilated left ureter.

A diagnosis of absence of the anterior abdominal muscles was made, and an attempt was made to relieve the urinary obstruction. A dorsal slit was made and following the passage of a sound a No. 3 Coude gumelastic catheter was passed and stitched in situ. A catheter specimen of urine was found to have a specific gravity of 1,002 and to be free of albumin. The blood urea level was $68 \mathrm{mg} .{ }^{\circ}$ 。

A retrograde cystogram showed gross dilatation of the bladder and reflux of dye up a markedly dilated left ureter (Fig. 4). A subcutaneous pyelogram confirmed the presence of a left-sided hydronephrosis. The right side appeared normal apart from spreading of the calyceal pattern.

The catheter was removed on the fourth day and the bladder was then emptied by manual compression.

On the seventeenth day the baby became febrile and examination of the urine showed a heavy Bact. coli infection. His general condition at this time was poor. The blood urea level was $92 \mathrm{mg}$. \%. His condition gradually deteriorated and he died aged 31 days. At necropsy the ureters and kidney pelves were grossly dilated and distended with infected purulent urine (Fig. 5). The urinary bladder appeared to be divided into an upper and a lower chamber separated by an opening which just admitted the tip of a little finger. The lower chamber was thought to be a dilated posterior urethra. No organic urethral obstruction was found.

Case 4. R.D. was first admitted to hospital in December, 1951, 24 hours after birth because of per- 


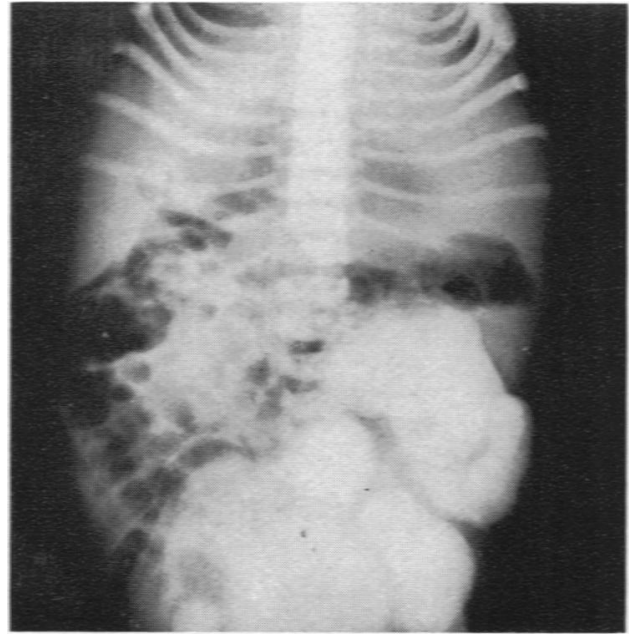

FIG. 4.-Retrograde cystogram in Case 3 to demonstrate reflux up the dilated and tortuous left ureter.

sistent cyanosis. He was the fourth child of healthy parents, the other children being free of abnormalities. His birth weight was $9 \frac{1}{2} \mathrm{lb}$. Examination showed a cyanosed infant, with a distended abdomen. The oblique and transversalis muscles were absent but the recti were present. Both testes were undescended.

He responded promptly to oxygen therapy and was discharged home.

Since then he had been re-admitted to hospital on at least 16 occasions because of recurrent respiratory infections. His latest chest radiographs in November, 1955, have suggested bilateral bronchiectasis. His present weight is only $34 \mathrm{lb}$.

Investigation has shown no abnormality of the urinary tract.

\section{Discussion}

The syndrome consists of either partial or complete absence of the anterior abdominal muscles associated with other congenital abnormalities. It occurs almost exclusively in males, although four cases have been recorded in females. The associated congenital abnormalities are chiefly of the genito-urinary tract, but numerous other anomalies have been noted. Amongst these are malrotation of the gut, talipes, cleft palate, spina bifida and hydrocephalus.

The commonest abnormal finding in the genitourinary tract is hypertrophy and dilatation of the bladder together with bilateral hydroureters and hydronephrosis. In spite of this evidence of urethral obstruction in many cases no organic lesion can be demonstrated. Several cases with an ectopic ureteric opening into a seminal vesicle have been reported, and Jameson and Cooper

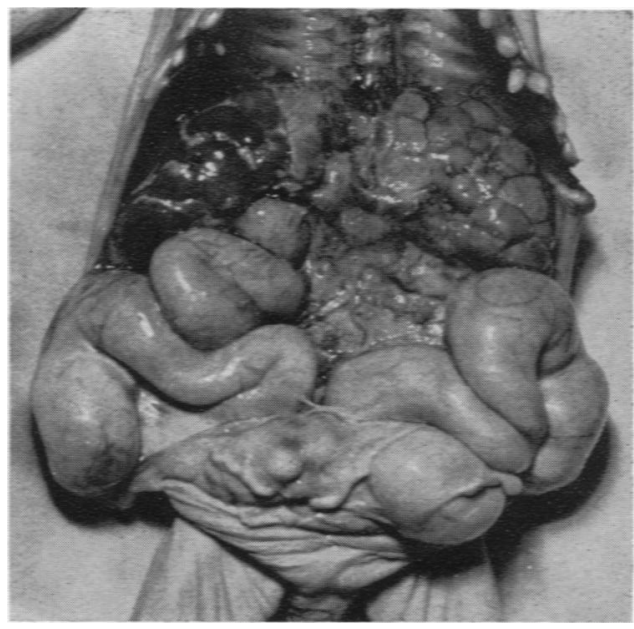

Fig. 5.-Appearance at necropsy in Case 3 showing the grossly hypertrophied and dilated ureters.

(1955) record a similar case in which the contralateral kidney was absent.

In males the testes are invariably bilaterally undescended. The condition is compatible with survival to adult life, but most cases die in infancy or early childhood.

The condition is recognizable at birth by the lax abdominal wall with lateral bulging and wrinkling of the skin (Figs. 2 and 3). The bulging of the abdominal wall is more marked on crying and straining. The abdominal organs are easily palpable and a dilated and hypertrophied urinary tract may be visible through the wall as in Case 3 .

The aetiology remains obscure, as it is undecided whether the dilatation of the renal tract is secondary to the absent abdominal muscles, or whether the muscles atrophy from pressure by the dilated bladder and ureters. In favour of the agenesis of the abdominal muscles being the primary defect is the fact that all cases of this condition which have been examined at necropsy have shown hypertrophy and dilatation of the bladder. Simple urinary obstruction with dilatation of the bladder, due to urethral valves or deformities of the bladder neck, is not associated with absent abdominal muscles. Housden (1934) has reviewed the various theories on the aetiology, and it can be said that little has been added to our knowledge since then.

The testes normally descend during the seventh month of intra-uterine life, but it is suggested that they are prevented from reaching the scrotum by the enlarged bladder and ureters.

The main danger to life is progressive renal 
failure from recurrent or persistent urinary infections aggravated by the severe degree of hydronephrosis.

In other children (Case 4), the main complication appears in the lungs. Here progressive and frequent respiratory infections lead to permanent structural damage. These cases are now being kept alive longer by the use of modern antibiotics.

Treatment consists in trying to prevent these infections, both urinary and respiratory. Frequent emptying of the bladder helps to prevent urinary stasis, and a well-fitting abdominal corset assists the deficient or absent abdominal muscles. In America surgical relief of the urinary tract abnormalities is being increasingly undertaken (Mathieu, Goldowsky, Chaset and Mathieu, 1953). Riparetti and Charnock (1954) report a case in which an attempt was made to strengthen the abdominal wall by fascial transplants and excision of loose redundant skin.

In those children prone to repeated respiratory infections early and energetic treatment will help to prevent the inevitable onset of irreversible structural lung changes.

\section{Summary}

Four cases of absence of the abdominal muscles are presented, in three of which there was gross demonstrable abnormality of the urinary tract.

The clinical condition and the present unsatisfactory state of our knowledge of the aetiology and the complications are discussed.

It is recommended that cases of this condition should have a full investigation of the genitourinary tract with a view to possible surgical treatment.

I wish to thank Dr. H. Everley Jones for permission to publish the first three cases and for helpful advice in the preparation of this paper. I also wish to thank Dr. J. V. C. Braithwaite for allowing me to include Case 4, and Mr. D. R. Paton for the photographs.

\section{REFERENCES}

Hill, A. (1947). Guy's Hosp. Rep., 9. 93.

Housden, L. G (1934) Archives of Disease in Childhood, 9, 219.

Jameson, S. G. and Cooper, J. O. (1955). J. Pediat., 47, 489.

Mathieu, B. J., Goldowsky, S., Chaset, N. and Mathieu, P. L. (1953). Ibid., 42, 92.

Riparetti. P. P. and Charnock, D. A. (1954). J. Urol. (Baltimore), 72. 541 . 